\title{
Modelling an ontology on accessible evacuation routes for emergencies
}

\author{
T. Onorati ${ }^{\mathrm{a}}$, A. Malizia ${ }^{\mathrm{b}}$, P. Diaz $^{\mathrm{a}}$ and I. Aedo ${ }^{\mathrm{a}}$ \\ ${ }^{a}$ Computer Science Dept., University Carlos III of Madrid, Avda. de la Universidad, 30, \\ 28911-Leganés, Madrid, Spain \\ ${ }^{b}$ Computer Science Dept.,, Brunel University, Kingstone Lane, \\ Uxbridge, UB8 3PH, United Kingdom
}

\begin{abstract}
Providing alert communication in emergency situations is vital to reduce the number of victims. However, this is a challenging goal for researchers and professionals due to the diverse pool of prospective users, e.g. people with disabilities as well as other vulnerable groups. Moreover, in the event of an emergency situation, many people could become vulnerable because of exceptional circumstances such as stress, an unknown environment or even visual impairment (e.g. fire causing smoke).Within this scope, a crucial activity is to notify affected people about safe places and available evacuation routes. In order to address this need, we propose to extend an ontology, called SEMA4A (Simple EMergency Alert 4 [for] All), developed in a previous work for managing knowledge about accessibility guidelines, emergency situations and communication technologies. In this paper, we introduce a semi-automatic technique for knowledge acquisition and modeling on accessible evacuation routes. We introduce a use case to show applications of the ontology and conclude with an evaluation involving several experts in evacuation procedures.
\end{abstract}

Keywords: Knowledge Management, Emergency Evacuation Route, Accessibility, Ontology Engineering

\section{Introduction}

When an emergency occurs or is going to occur, affected people need to know the location of safe places where they can shelter and routes for reaching them. In this way, depending on their abilities and the surrounding environment, users can be guided along such routes reducing the number of victims. Within this scope, the preparedness phase has a crucial role in emergency management for defining an effective evacuation route diagram. Generally, depending on the area of interest, there is a security department in charge managing the evacuation plan, where, not only routes, but also procedures and responsibilities are identified. A support for this activity consists of simulations, guidelines, mathematical models, regulations and best practices that could help in the implementation of a valid evacuation route diagram.

In order to guarantee an effective evacuation for all people affected by a disaster, we think that a route diagram must include additional variables, such as user's profile (e.g. functional or contextual disabilities, elderly, and children), kind of emergency (e.g. typhoon, earthquake, and tornado) and any other exceptional circumstances (e.g. interrupted roads, collapsed exit). For example, when an emergency occurs in a building, a person with limited motor abilities needs a special route for escaping with the help of an assistant. Moreover, in this case the route cannot pass through stairs or narrow passages.

To make routes accessible for every user and emergency circumstance, it is crucial that tools used for the definition of the diagram acquire and share this information with other emergency management 
systems. For this reason, a standard for managing and distributing knowledge about accessible evacuation routes is needed as support for this activity.

The aim of this paper is to define a knowledge representation for providing adapted information about evacuation routes depending on emergency scenarios, user's profile and communication technologies. The proposed solution is based on a previous work (Malizia et al., 2010), where authors have developed an ontology called SEMA4A (Simple EMergency Alerts 4 [for] All). SEMA4A reflects the necessity of an interoperable knowledge for relating concepts about accessibility guidelines, emergency scenario and communication technologies for providing accessible notifications. The novelties introduce in this work can be summarized as follows: (1) giving to users an active role during the response phase by guiding them to a safety point (our previous work included only notifications about emergencies but no active guidance to escaping routes); (2) the extension of the ontology for including evacuation routes is performed introducing methodologies for semi-automatic knowledge identification, acquisition and modeling; (3) an use case (fire scenario) with the application of new technologies related to the new requirements demonstrating the applicability of the approach.

The extended version of SEMA4A aims at notifying users with, not only information about the specific situation, but also personalized instructions for reacting effectively and reaching the nearest safety point. The second contribution consists in applying an advanced methodology (with respect to the first version of SEMA4A) due to the dynamic characteristics of the knowledge base modeled for evacuation. Following the Knowledge Management life cycle (Maier, 2007), this methodology covers different steps: from knowledge identification to the organization through acquisition and creation. Moreover, in order to ensure an interoperable knowledge representation, the ontology has been implemented using the Web Ontology Language (OWL). Through this standard codification we are providing an open knowledge base for the integration with other systems and tools.

In order to show the applicability of SEMA4A, we have collaborated at the development of a prototype for sending notifications about evacuation routes. In such prototype not only the evacuation route, but also the visualization mode, is adapted considering the particular user's profile, the characteristics of the situation and using new interaction paradigms like augmented reality.

Finally, in order to show the validity of the proposed extension, we have evaluated it employing two different approaches: quantitative and qualitative. Results obtained from both methodologies demonstrate that the ontology has a high representative value for considered domains and consequently can be used as an interoperable base for adapting information about evacuation procedures.

In the second section, we present a summary of literature contributions about accessibility, emergency and evacuation models and systems. Successively, in the third section we introduce the proposed extension, specifying the applied methodology and describing the applicability of obtained result through a use case. In the fourth section, we present evaluation results for quantitative and qualitative evaluation. Finally, some conclusions and future works are discussed.

\section{Background}

\subsection{Knowledge Representations for Accessibility and Emergency}

In literature, several interesting contributions have been introduced to define knowledge representations as a common and formal language to enable interoperability of different services. Focusing on the domain of accessibility, Yesilada et al. have developed a tool called Dante for making accessible web pages for people with visual impairments (Yesilada et al., 2004). Dante is based on an ontology called WAfA (Web Authoring for Accessibility) that contains concepts and relations that model the structure and navigation of web pages. Through WAfA, the proposed system provides annotations and tags for each element of a web page. In this way, a web page is made accessible for any reader and any users' profile.

Following the same idea, Lozano-Tello et al. have published the KAICO system that associates semantic tags to web pages in order to extract and communicate information to blind people (Lozano-Tello et al., 
2004). The system uses an ontology called OntoSaw for establishing a correlation between concepts on web pages and concepts on the accessibility domain. In this way, it is possible to determine if elements contained in a web page are accessible or not. The ontology has been defined starting from several interviews with people with visual impairments about their experience surfing the web.

Other interesting examples of knowledge representations can be also found in the emergency domain. Most relevant contributions are focused on the response phase and in particular on the lack of a common language among different organizations and agencies. One of them is the Emergency Response Ontology by $\mathrm{Li}$ et al. in 2008 ( $\mathrm{Li}$ et al., 2008). This ontology is a semantic representation of the emergency response workflow. Based on it, information systems for managing crisis situations can infer the next action to perform (according to defined procedures) and who is in charge of its execution.

Considering that we aim at adapting information about evacuation procedures to several variables, i.e. accessibility, emergency, technology and evacuation, we need to model an integrated representation of four different domains, obtained extending the SEMA4A ontology. On the contrary, previous contributions found in literature focus mainly on single aspects, like the users' profile (e.g. the OntoSaw ontology) or the emergency response (e.g. the Emergency Response Ontology).

\subsection{The SEMA4A ontology}

Providing alert notifications about current emergencies is crucial for reducing the number of victims and damages. In order to reach all affected people in every kind of situation, it is important to consider the users' needs and communication infrastructures available depending on the particular emergency. In 2010, the SEMA4A (Simple EMergency Alerts 4 [for] All) ontology was developed for correlating users' needs, technologies and relevant information about emergency situations (Malizia et al., 2010). This ontology can be used by emergency notification systems as support for adapting, communicating and sharing information among different sources and platforms.

Information included in SEMA4A is categorized into three main classes: (1) Accessibility; (2) Emergency; (3) Communication. The Accessibility class collects terms from two existing ontologies: the Web Authoring for Accessibility (WAfA), about the structural organization and navigation of web pages, and the AccessOnto ontology, related to accessibility requirements and in particular to guidelines defined by the Web Accessibility Initiative, Sun Micro Systems, IBM, Microsoft and Apple. SEMA4A's second class, Emergency, together with the third one, Communication, include terms and relations about emergency and media technologies, extracted applying a semi-automatic technique that takes in input an existing taxonomy and the general purpose ontology WordNet (Miller et al., 1990).

In order to validate collected terms and relations, SEMA4A was evaluated both quantitatively and qualitatively (Malizia et al., 2010). The aim of the quantitative evaluation was to calculate two measures, such as coverage and accuracy, over a corpus of documents, to understand if the ontology was representative for selected domains. The qualitative evaluation was performed to value its usefulness, proposing a short questionnaire to two experts of accessibility and emergency management. Successively, results obtained from evaluators have been used to improve SEMA4A.

Another interesting characteristic of SEMA4A, that facilitates the interoperability with other platforms is the usage of $\mathrm{OWL}$, a standard markup language for codifying collected knowledge through a formal semantics. Moreover, the consistency of its classes was verified with a reasoning tool called Pellet developed by Mindswap laboratory at Maryland University.

Finally, same authors have demonstrated the applicability of SEMA4A developing a prototype called CAP-ONES (Common Alerting Protocol-based Open Notification System) and presented in (Malizia et al., 2009). The aim of CAP-ONES was to provide people with notifications about emergencies, personalizing them depending on users' abilities, characteristics of the situation and used technologies. For example, by performing a query over concepts and relations included in SEMA4A, the prototype could determine which kind of media was more appropriate for sending messages, considering the current situation (e.g. earthquake, tornado, ...) and users to reach (e.g. elderly, disabled, ...). The query is executed using SPARQL, a W3C (i.e. World Wide Web Consortium) recommendation that allows inferring information 
from a RDF (i.e. Resource Description Framework) document. Figure 1 shows an example of query executed over SEMA4A for determining the list of channels and media that can be used for communicating during any kind of emergency situations. In particular, obtained channels and media (i.e. ?mu in Figure 1) are selected depending on the supported content (i.e. ?emgmusc :can-communicate ?mu in Figure 1) that can be used for the emergency notifications (i.e. ?emg :mayUse ?emgmu; ?emgmu rdfs:subClassOf ?emgmusc in Figure 1).

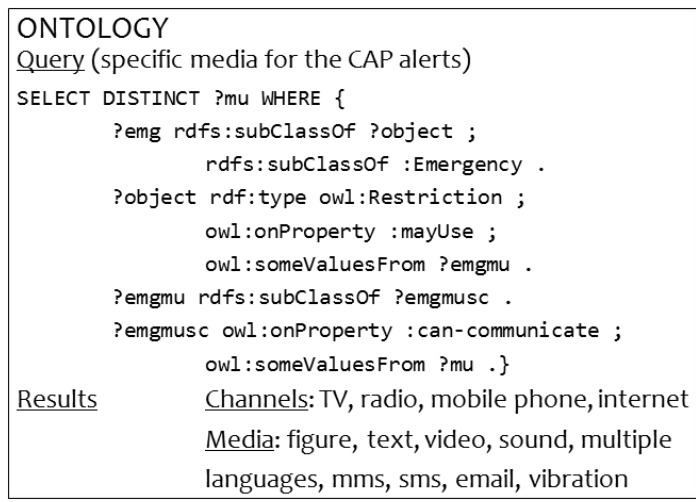

Figure 1. Example of a SPARQL query executed over SEMA4A by the CAP-ONES prototype.

The scope of the SEMA4A ontology focuses on the notifications of emergency alerts. Nevertheless, the successive step after users have been notified about what is going on would be to provide them with information about how to react to the potential danger. If they are in a dangerous area, they have to escape as soon as possible and reach a safety point. If not, they must also be aware of their location to avoid potential dangers.

\subsection{Emergency Evacuation Models and Systems}

When an emergency occurs or is going to occur, one crucial task for helping affected people is notifying them about which routes can be used for escaping. In order to ensure that everybody can reach a safe place, it is important to take into account not only characteristics of the environment but also users' needs, such as: contextual or functional disabilities, elderly, social and individual behaviors in emergency situations.

In literature, both strategies and mathematical models have been defined with the common aim of finding an optimal and fast solution for the evacuation problem. In (Jeongin et al., 2013), authors revisited several strategies for evacuating a high-rise building identifying the most appropriate situation to use it. For example, elevators can be used for people with wheelchairs as an effective evacuation way, but also for avoiding any possible delay for the other people.

Considering the area to evacuate as a network, several algorithms have been implemented with constraints like dimension, number of evacuees and possible bottlenecks. In (Kim et al., 2007), authors have categorized available algorithms into three main categories: (a) Linear Programming; (b) Simulation methods; (c) Heuristic methods. The difference among such categories lies in the objective they aim to reach. In case of linear programming, methods try to minimize the overall evacuation time looking for an optimal route diagram. Simulation methods are based on observing the behaviors of individuals during an evacuation procedure and trying to predict them. Finally, the aim of heuristic methods is related to reduce the computational cost combing and extending available algorithms, as in (Yamada, 1996).

By analyzing these contributions, we can find several common aspects. As stated in (Hamacher and Tjandra, 2001), methods for implementing effective evacuation route diagrams could differ in proposed objectives or applied algorithms, but all share the need for a set of basic inputs, as summarized in the following list: 
- location, both indoor and outdoor, such as buildings, stadiums or whole cities;

- people's behaviors;

- user's profiles, focusing in particular on special needs, assistants and supports;

- type of emergency and affected areas;

- $\quad$ safe places for people at risk;

- emergency workers and organizations.

This information is also reflected in standard guidelines for developing evacuation route diagrams, defined by governmental and non-governmental agencies. For example, the ASTM (American Society for Testing and Materials) has collected several guidelines about the appropriate terminology to use, the content and the format for preparing routes to escape from a building. Another interesting contribution is represented by facilities provided by the OSHA (Occupational Safety and Health Administration) to implement efficient evacuation plans and procedures, focusing in particular on assigning escaping routes.

In order to develop effective evacuation routes diagrams, safety and security departments generally apply standard guidelines and mathematical models, specifying a set of input data, as mentioned above. This initial step consists in customize routes combining characteristics of the emergency, user's profile and evacuation information.

In literature, there are several contributions on the development of decision support systems on how to design an effective evacuation plan for a specific area. In (Manley and Kim, 2012), the authors propose a model that takes advantages of different evacuation strategies in order to determine the most appropriate one depending on the characteristics of the environment and the affected people. This model focuses in particular on the most vulnerable groups and represents them in terms of abilities and speed for reaching a specific point. Moreover, in this way it is possible to find and consequently solve any possible bottleneck in the evacuation plan.

Another interesting contribution about planning and decision making of an effective evacuation is a prototype presented in (Wagner and Agrawal, 2014). The prototype is a decision support system that uses an agent-based model for simulating the evacuation of a large number of people from an indoor building in case of a fire event.

Once the evacuation plan and procedures have been defined, instructions about the routes for escaping are notified to affected users. Several systems have been developed to make this task more efficient. To understand how such systems deal with personalization and accessibility of evacuation routes, we have reviewed several of them, as listed in Table 1. In particular, the systems analysis focuses on notifications, personalization and accessibility of evacuation routes.

\begin{tabular}{|c|c|c|c|c|}
\hline System & Reference & Notification & $\begin{array}{l}\text { Personaliz } \\
\text { ed } \\
\text { Route }\end{array}$ & $\begin{array}{l}\text { Accessibili } \\
\text { ty }\end{array}$ \\
\hline CodeRed & www.ecnetwork.com/codered & Phone call & Yes & No \\
\hline $\begin{array}{l}\text { The Digital } \\
\text { Building }\end{array}$ & archaio.com.concentric.com/emsolution.html & No & Yes & No \\
\hline Huens & $\begin{array}{l}\text { www.uos.harvard.edu/opscenter/emergency } \\
\text { _management.shtml }\end{array}$ & $\begin{array}{l}\text { Social } \\
\text { networks, sms, } \\
\text { text and voice } \\
\text { emails }\end{array}$ & No & No \\
\hline Cucem & www.cucems.com/index.html & Radio, TV & No & No \\
\hline cAlert & calert.uchicago.edu & $\begin{array}{l}\text { Emails, phone } \\
\text { calls, sms }\end{array}$ & No & No \\
\hline $\begin{array}{l}\text { Inoues's } \\
\text { system }\end{array}$ & (Inoues et al., 2008) & $\begin{array}{l}\text { Mobile } \\
\text { application }\end{array}$ & Yes & Yes \\
\hline
\end{tabular}

Table 1.Emergency Notification and Evacuation Systems and their main features extracted from state of the art. 
Considering accessibility features, among reviewed systems, just the Indoor Emergency Evacuation Service developed by Inoue et al. (2008) provides a sort of very basic accessible mechanism sending the notifications both as textual description and audio recording. Once the message has been received, the user can choose one of these two modalities to access to the instructions.

Concerning personalization, while CUCEM and cAlert notifications just provide general information about evacuation procedures (location of shelters, available exits or meeting points), other systems also provide each person with ad-hoc instructions about how to reach the nearest safe place depending on his/her location. Nevertheless, this is not enough to ensure an efficient evacuation for all affected people: other variables need to be taken into account, i.e. users' abilities, exceptional circumstances and devices used for receiving notifications or visualizing routes. For such reason, in this work we propose to extend the SEMA4A ontology integrating a new set of concepts and relations specific for evacuation routes. In this way, we can define an interoperable knowledge representation that can be accessed by different systems for extracting contextual information and providing personal and accessible evacuation routes.

\section{Modeling accessible evacuation routes: the extension of SEMA4A ontology}

In order to adequately represent information about evacuation routes, adapting it to users' preferences and characteristics of the emergency, we propose to extend SEMA4A ontology. With respect to the development process adopted for SEMA4A's previous version, in this case we could not find any taxonomies or existing knowledge representation about evacuation procedures and plans. Consequently, we have collected a set of documents called corpus. The corpus has been chosen considering the kind of information we wanted to conceptualize. In fact, when an emergency occurs it is crucial to know official plans from the emergency departments or agencies as well as specific characteristics of the event. For example, during the Japanese tsunami in March 2011 the best way for communicating with affected people turned out to be the loudspeakers. This information has to be included into the ontology as part of the available communication infrastructures not damaged by the tsunami. For this reason, we included in the corpus documents about official evacuation plans, adaptation for vulnerable groups and emergency characteristics focusing on potential communication channels that could be damaged. Moreover, all documents included in the corpus are available online and they may change over time dynamically including new relevant details.

Collected documents have been analyzed looking for sentences that can be considered relevant for our scope (e.g. "an evacuation plan contains a set of routes"). From these sentences, both nouns and verbs have been extracted as candidate to be included as concepts and relations in the SEMA4A ontology. In the example provided above, concepts would be "evacuation", "plan" and "route", and relations among them would be defined by two triples <evacuation, contains, route $>$ and $<p l a n$, contains, route $>$. These activities are quite difficult to perform manually. For this reason, we have tried to automate the entire process applying well known techniques in the area of Ontological Engineering. Consequently, it is crucial to verify the validity of obtained triples with a a qualitative evaluation involving several domain experts. By interviewing experts about which triples to include into the ontology we have received suggestions and comments for improving their representativeness.

This methodology has been inspired by the Knowledge Management Life Cycle introduced by Maier (Maier, 2007). As shown in Figure 2 and detailed in the following sections, the design process covers four steps: identification, acquisition, creation and organization. 


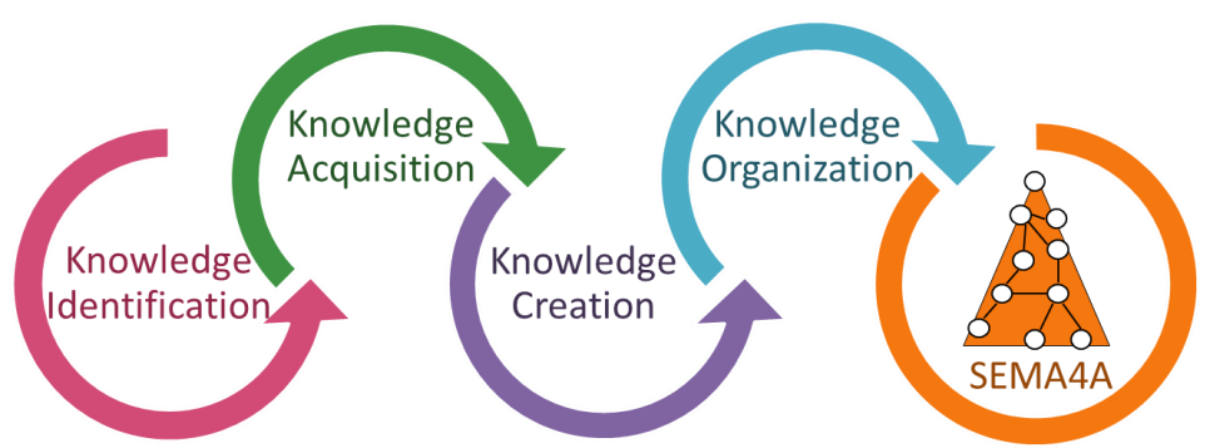

Figure 2. The four steps of the design process for extending the SEMA4A ontology.

\subsection{Knowledge Identification}

The aim of the first step, called Knowledge Identification, is to identify a set of sources (i.e. corpus) for retrieving relevant knowledge. Such sources could be both internal and external with respect to the considered domain. Within the scope of this work, we have collected almost one hundred documents: both research contributions (i.e. books, papers or magazines) about evacuation models and simulations, and reports published by governmental organizations and emergency management institutions such as guidelines and plans. These documents have been chosen considering our scope, that is to model a representation of statements like "walk down the aisle" or "take the first road on the right" that contain specific terms ofcivil engineering domain which ae strictly related to evacuations. Moreover, all documents and terms are available on the Web and they could change over time so that results obtained from applying such methodology could differ dynamically.

Successively, after identifying document and concepts sources, next tasks will consist in the acquisition and organization of such concepts.

\subsection{Knowledge Acquisition}

In the second step, called Knowledge Acquisition, previous information has been acquired and modeled in our ontology. In particular, the collected corpus has been analyzed tagging each word with its semantic function (e.g. nouns, verbs and adjectives) to find and extract a list of about 5,250 nouns as possible candidates for the ontology. At this point, not all terms in the list are significant as knowledge representation for the domain of evacuation routes. In order to identify the most relevant ones, we have filtered them experimentally combining two semantic functions: Domain Relevance and Domain Consensus, both defined in (Navigli et al., 2003).

The Domain Relevance compares the frequencies of terms among different domains in order to determine if they are specific for a particular area. From the analysis of collected documents, we have recognized several contributions in the area of civil engineering, architecture and emergency mainly due to descriptions of buildings, locations and risk management. For this reason, civil engineering, architecture and emergency have been chosen as contrast domains for computing the Domain Relevance. In particular, for representing adequately the civil engineering and architecture domains, we have collected several handbooks from GoogleBooks ${ }^{1}$. For the emergency domain, we have considered the same corpus used for the quantitative evaluation of SEMA4A (Malizia et al., 2010). This corpus consists of several research contributions already published in the Proceedings of the International Conference on Information Systems for Crisis Response and Management (ISCRAM). Given the four domains: evacuation routes (D1), civil engineering (D2), architecture (D3) and emergency (D4), the Domain Relevance of a term $\mathrm{t}$ in D1 is formally defined as

$$
D R_{t, D_{1}}=\frac{P\left(t \mid D_{1}\right)}{\sum_{j=1}^{4} P\left(t \mid D_{j}\right)^{\prime}}
$$

${ }^{1}$ http://books.google.com/ 
Where $P\left(t \mid D_{k}\right)=\frac{f_{t, k}}{\sum_{t \prime \in D_{k}} f_{t^{\prime}, k}}$ with $k \in\{1,2,3,4\}$.

By comparing the probabilities of terms considered in each domain, we obtain that terms with a high value of Domain Relevance are most significant for the domain of evacuation routes.

The aim of the Domain Consensus is to identify which terms are most frequent among all documents in the corpus in order to identify most representative ones for a specific domain. We needed to compute and successively combine the frequency of each term in each document through the entropy function (H). Given the domain of evacuation routes (D1), the Domain Consensus of a term $t$ is formally defined as

$$
D C_{t, 1}=H(P(t, d))
$$

Where $H(P(t, d))=\sum_{d \in D_{1}} P(t \mid d) \log \left(\frac{1}{P(t \mid d)}\right)$ and $P(t \mid d)=\frac{f_{t, d}}{\sum_{d \in D_{1}} f_{t, d}}$.

In order to identify a list of relevant terms, results obtained from computing Domain Relevance and Domain Consensus have been combined in Term Weight (TW) formally defined as:

$$
T W_{t, 1}=\alpha D R_{t, 1}+(1-\alpha) D C_{t, 1}
$$

Where the parameter $\alpha$ is in the range $[0,1]$. To determine it, we have compared the rankings of terms obtained for different values of $\alpha$ and looked at their relevance with respect to the domain of evacuation. As result, the parameter has been set experimentally to 0.75 by looking at terms with highest values and verifying their relevance in the domain.

The term with the highest TW for $\alpha=0.75$ is evacuation with 1.92 , which is highly relevant for the considered domain of interest. It is interesting to note that the highest value for the Domain Relevance (i.e. 1,00 ) has been reached by terms only partially relevant for the domain as stairway, pager and auditorium, while evacuation has a 0.96. In the same way, the highest Domain Consensus (i.e. 5.01) has been obtained for the term building, while evacuation has a 4.8 score. This fact confirms that to determine adequately the relevance of a term a combination of the two functions is needed.

\subsection{Knowledge Creation}

In the third step, Knowledge Creation, we performed tasks for the creation of previously acquired knowledge. The creation phase is strictly related to the individual and collective learning process for the specific domain of interest..

In this case, we have defined a mapping among the knowledge acquired for the evacuation domain. We used a natural language processing technique. This technique uses the same corpus of documents of the Knowledge Identification phase successively tagged by a POS (Part Of Speech) tagger ${ }^{2}$ during the Knowledge Acquisition phase. From the tagged corpus, several triples composed by two nouns and a verb in their root forms were extracted. These triples are possible candidates for the mapping, where two nouns are terms already acquired for the domains of interest and the verb is the relation among them. We obtained 20,161 candidates, successively filtered manually looking for any inconsistencies or redundancies taking into account the relations already included in the knowledge representation. Moreover, for identifying the most representative relations we used Term Weight values computed in the previous phase.

\subsection{Knowledge Organization}

After the knowledge creation, the fourth step, called Knowledge Organization, consisted of linking new terms about evacuation routes not only among them but also with the previous ontology. To do this, we looked at terms co-occurrences in the collected corpus obtaining several relations as triples composed

\footnotetext{
${ }^{2}$ A POS tagger is an algorithm for labeling a word in a text (corpus) as corresponding to a particular part of speech, such as: noun, verb, adjective, etc.
} 
by a verb in its root form and a couple of terms. In this way, we are not only organizing knowledge about evacuation but also integrating it with the previous ones included in SEMA4A about emergency, technology and accessibility.

The Evacuation class, introduced in the updated version of SEMA4A, is a structured representation of terms defined from scratch about evacuation procedures, standards and models. During the entire design process, we have noticed that the domain of evacuation is strictly related to other domains, like architecture and civil engineering. As consequence of this idea, the Evacuation class has been organized into four subclasses (see Figure 3):

- the Location class concerns all terms we have collected about the physical environment in which an evacuation procedure is defined (e.g. stairs, building, elevator);

- the Personnel class represents people involved in the evacuation, focusing in particular on their role (e.g. supervisor, visitor, employer);

- the Procedure class includes technical terms about standard procedures or plans (e.g. instruction, kit, point);

- The Transportation class is related to the different means that can be required during an evacuation for escaping (e.g. bus, car, congestion).

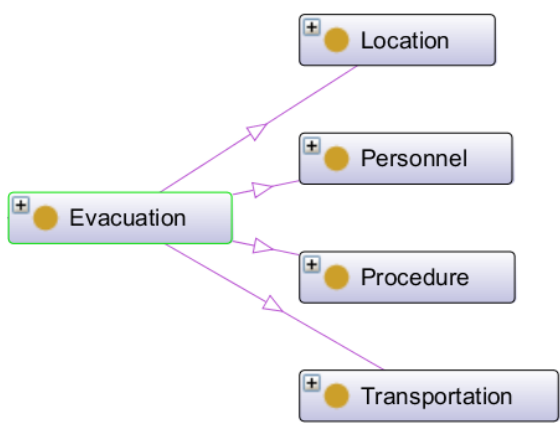

Figure 3.The main structure of the Evacuation class.

\section{Use Case}

In order to show the applicability of SEMA4A, here we present a scenario of use of a notification system developed for sending personalized evacuation routes in indoor environments through mobile devices (Aedo et al., 2012).This system executes several queries over SEMA4A in order to guarantee the personalization of evacuation routes depending on several variables: user's abilities or impairments, levels of expertise on the surrounding environment (e.g. an employee might be familiar with a building while a visitor might easily get lost), needs or preferences, current location, media and technology being available, characteristics of the surrounding environment and emergency context (e.g. severity and evolution). It is important to clarify that people needing special assistance during an emergency situation are, not only those that suffer from permanent disabilities, but also other groups with contextual disabilities (e.g. during fire smoke will prevent from seeing all people). Within these groups, we consider in particular people unfamiliar with the building structure (e.g. visitors) or with limitations due to the event (e.g. fire smoke).

The system architecture is composed by three prototypes (see Figure 4): (A) CAP-ONES adapts the notifications to user's profile and emergency characteristics; (B) iNERES (i.e. i (mobile) Notification of Evacuation Route in Emergency Situations) retrieves the position of the user; (C) NERES (i.e. Notification of Evacuation Route in Emergency Situations receives the user's position from iNERES, selects from the official evacuation plan the most suitable route considering the results obtained from querying SEMA4A, and finally sends it to iNERES. Not only the evacuation route, but also the way to visualize it in the application is adapted to the user's profile choosing among textual description, 3D image sequence, map and augmented reality. 


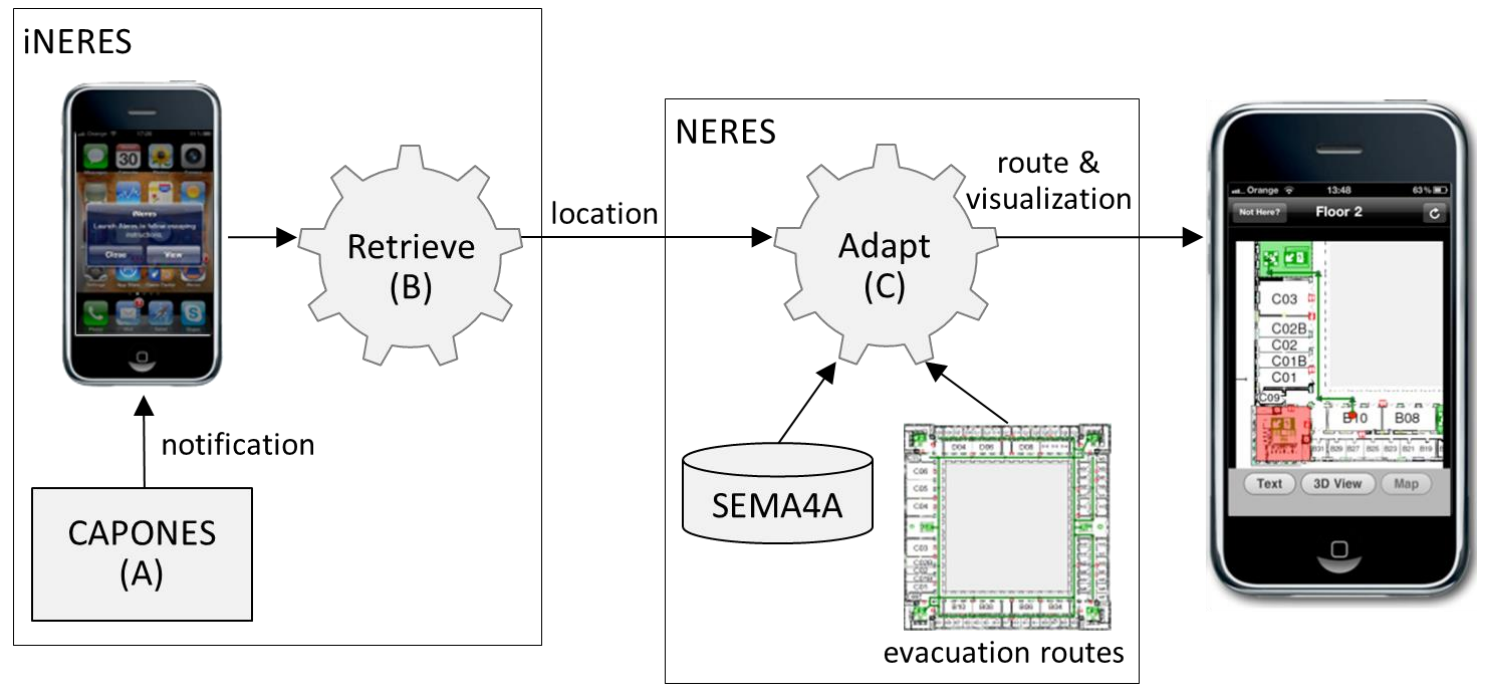

Figure 4. The system architecture for sending personalized evacuation routes

The iNERES application has been also evaluated simulating the evacuation of a university building after a real fire occurred in a laboratory. Involved people were twelve graduated students, ten males and two females, all of them between 18 and 35 years old. They have been selected because not familiar with the building and not aware of exits and safe points. For this reason, they were considered as part of a vulnerable group (e.g. including among others people in wheelchair or visual impaired for fire smoke) and consequently their smartphones were provided with the iNERES application.

Each user was located in an office close to the laboratory where the fire occurred. When evacuation started, users received a notification through the iNERES application with detailed information about the situation and the instructions to follow for reaching safety point (see Figure 7a). Such information was adapted to each user's specific needs, including the location and any damages caused by the fire. To do this, the NERES module performed the following queries over SEMA4A:

- Since the user is not familiar with the building where her office is, she can be considered as vulnerable as one with limited abilities due to his particular context (i.e. contextual disability). In SEMA4A, this is represented as a Novice level of Expertise on the surrounding environment (e.g. knowledge of building pathways and structure) having difficulties in navigating the map of the floor and orientating herself in reaching the exit. From the proposed ontology a system might infer that people with Novice Expertise may use a non-conventional interaction tool, such as map zooming or augmented reality view for the evacuation route (see Figure 5). Consequently, the NERES system can selectfrom our ontology the augmented reality overlapping directions to follow to the actual camera view or displaying points of interest on the images coming from the phone camera (see Figure $7 \mathrm{~b}$ ). In this way, the user is not required to have previous knowledge on the surrounding environment in order to follow the instructions. This is valid in particular when the visibility is reduced by external agents as the smoke (the instructions can be sent in textual form for a Mobile device equipped with text-to-speech reader). 


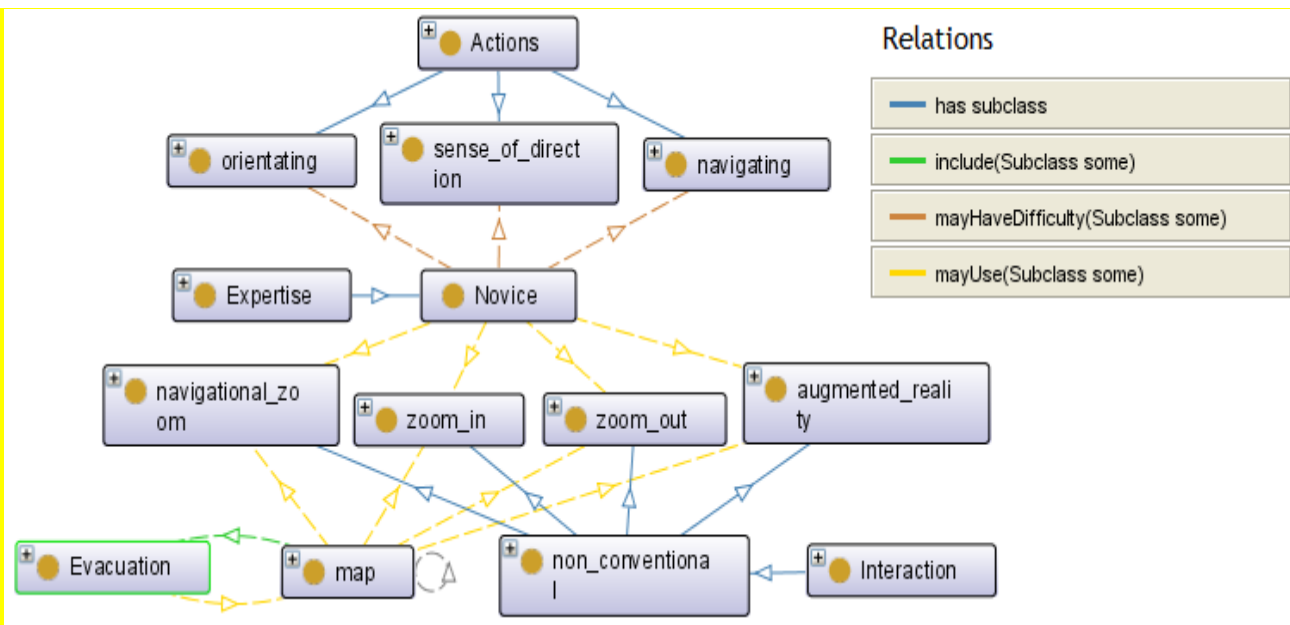

Figure 5. The conceptualization of Novice Expertise obtained from the SEMA4A ontology.

- In order to ensure that each person involved in the simulation could find the notified information useful for escaping and reaching a safety point, NERES had also to infer from SEMA4A the Web accessibility guidelines specific for the Novice Expertise (see Figure 6). The queried guidelines have been collected from the AccessOnto ontology (Malizia et al., 2010). They refer to an effective and minimalist learning process (CG_24 and CG_25) and a minimum number of steps to perform for completing the tasks and making decisions (CG_26).

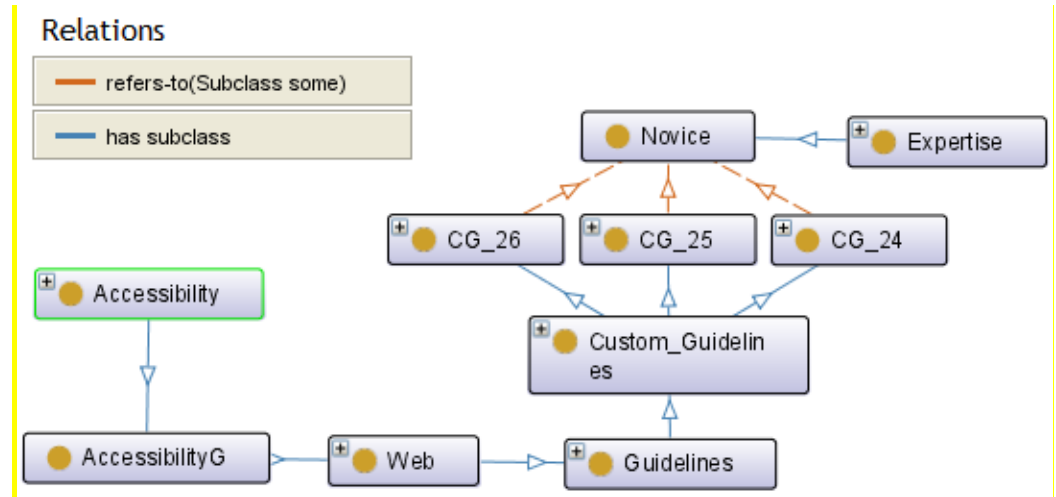

Figure 6.The conceptualization of the Web accessibility guidelines for Novice Expertise obtained from the SEMA4A ontology.

Taking into account the results obtained executing the queries over SEMA4A, the participants at the simulation received a short but effective description of the steps to follow for escaping safely from the office where they are. Moreover, such instructions are shown with an augmented reality view by superimposing arrows and signs to the device's camera view (see Figure $7 \mathrm{~b}$ ).

In order to show how SEMA4A can be used in a more dynamic scenario, we have simulated damages along the suggested route. In this way, the users had to stop following the instructions and communicate the interruption to the operators in charge of managing the evacuation. At this point, the NERES module recalculated the evacuation routes adding the new information to the performed queries. In particular, the corridors interrupted by damages can be avoided inferring from SEMA4A the relation <route, cannot contain, damage>.

After the simulation, the participants were asked to answer to a questionnaire about the usability of iNERES receiving very positive results. They considered the application easy to learn and to use, in particular thanks to the usage of a clear terminology for the instructions. Concerning the provided functionalities, they found them helpful if an evacuation is needed during a critical situation. They also 
identified some problems with the performance of the system due to a not well-working Internet connection in the building.

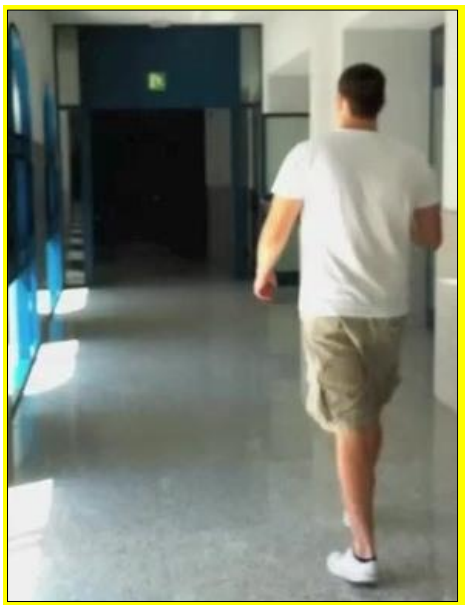

(a) An user following the route

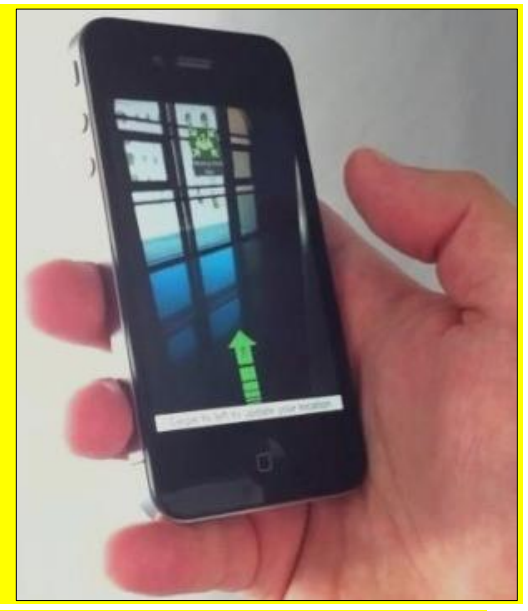

(b) The augmented reality view

Figure 7. The simulation performed with the iNERES application.

\section{Evaluation}

In literature, there are several contributions about quantitative and qualitative methods for evaluating ontologies. In this paper, we focus on a quantitative technique inspired by (Spyns and Reinberger, 2005) and (Zipf, 1949) and a qualitative technique inspired by the evaluation phase of the HCOME methodology (Kotis and Vouros, 2005). The HCOME methodology aims at actively involving domain experts communities within the whole ontology life cycle: from development to evaluation phase.

\subsection{Quantitative Evaluation}

The main purpose of the quantitative evaluation is to demonstrate the validity of identified terms and relations as candidates for extending SEMA4A ontology. In (Malizia et al., 2010), authors presented an evaluation method based on the definition of a set of triples and a corpus of documents. Triples are lemmas composed by two terms and a role that co-occur in the ontology. Successively, three measures (coverage, accuracy and precision) are computed in order to determine how much lemmas are representative for the proposed corpus.

During the extraction methodology, we collected lemmas as candidate relations and we evaluated their validity over the collected corpus of documents. Following the method of Spyns and Reinberger, called EvaLexon, we measured the validity of considered lemmas through two lexical criteria: coverage and accuracy (Spyns and Reinberger, 2005). Both coverage and accuracy are measured grouping lemmas in frequency classes: each class includes lemmas with the same frequency. Coverage represents how many lemmas are representative for the domain and it is measured counting for each frequency class, which terms of the lemmas belong also to the corpus. Accuracy indicates the specificity of retrieved terms with respect to the selected domain. Each frequency class includes all the lemmas with the same frequency value over the corpus of documents.

In our case, we have collected 362 classes, where the lower lemma occurs just one time and the higher has a frequency of 5,818. For coverage, the obtained ratio between the number of lemmas that are also in SEMA4A and the total number of lemmas in the corpus is $58.9 \%$. In order to determine the accuracy, we needed to select a specific range of classes i.e. the most relevant for representing the evacuation domain. To do this, we applied the Zipf's law. In (Zipf, 1949), the author clarifies the relation between the frequency of a word and its relevance for a domain: terms in higher frequency classes are general and less specific, as for example stop words. The same theory concerns classes with a lower frequency. 
As a consequence, most significant terms for a domain can be found in the middle classes, as stated in (Luhn, 1958).

Taking into account the Zipf's law, the selection of most relevant classes has been done manually: we looked at meanings of each term. In this case, we used the already computed domain relevance $D R_{t, D_{1}}$ as support for this step and we have determined for each frequency class the ratio of relevant terms. As final result, we have identified the following categories, where FC stands for frequency class:

- $\quad F C<28$, general words not directly related to the domain (e.g. "proximity" with frequency 25);

- $28 \leq \mathrm{FC}<500$, general words that acquire a specific meaning if used in the domain (e.g. "access" with frequency 241);

- $\quad F C \geq 500$, specific words for the domain due to the usage of technical reports among the corpus of documents (e.g. "evacuation" with frequency 5818).

With respect to the theory of Luhn (Luhn, 1958), we have found most specific terms for the domain in the higher classes. This fact is related to the kind of documents we have collected in the corpus: all of them are quite specific and written with a technical language, nonetheless when modeling evacuation routes these kinds of terms are generally very used.

Most relevant terms for the domain have been identified starting from the frequency class 28 . On average, the accuracy ratio for selected range is $64.2 \%$, a quite high improvement with respect to the coverage ratio (58.9\%). From a lexical point of view, we have obtained a very precise result probably due, not only to used corpus, but also to the novel methodology we have adopted for the term extraction. Figure 8 shows a graphic representation of the accuracy: for a more readable image, we have cut off frequency classes higher than 300. In the graph, the frequency classes are on the horizontal axis, while the number of lemmas contained in each one of them is on the vertical axis. The brown part and the pink part are the distribution of the lemmas contained, respectively, in the corpus and in the ontology over the frequency classes. Graphically, the accuracy is represented by the overlapping between the two parts: the brown and the pink.

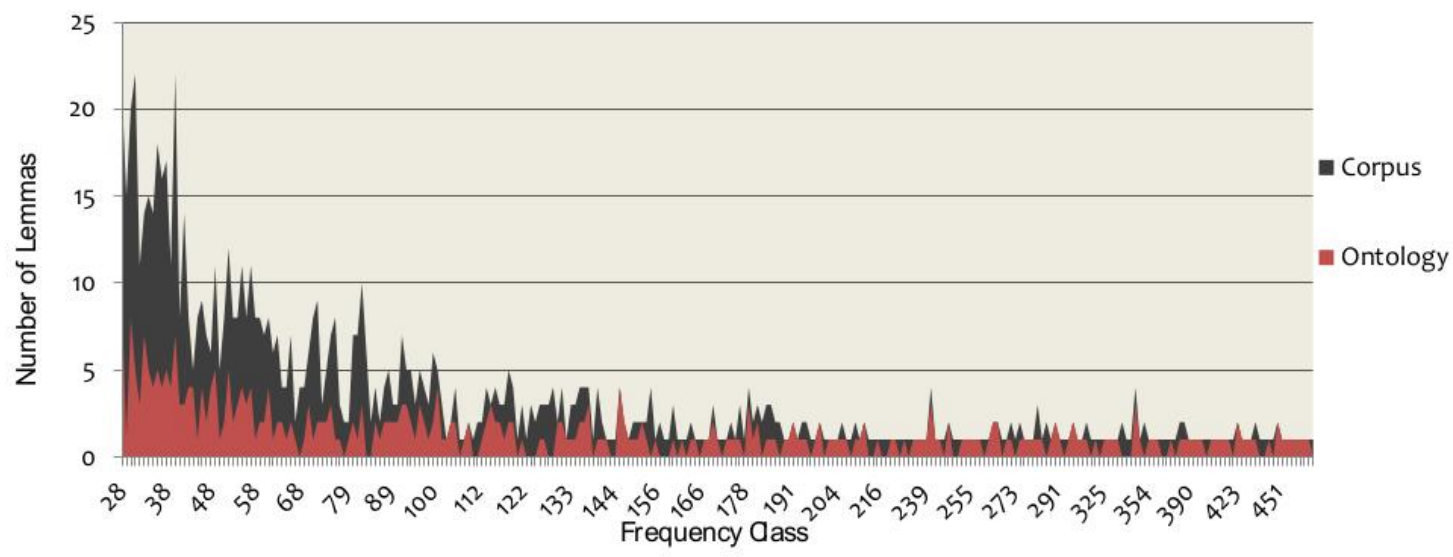

Figure 8. Accuracy of the ontology over the corpus

\subsection{Qualitative Evaluation}

Inspired by the HCOME methodology for increasing the participation of knowledge workers (Kotis and Vouros, 2005), the qualitative evaluation aims at involving domain experts for determining how many collected terms and relations are representative for their area of expertise.

In this work, evaluators were five international experts about evacuation procedures:

- three engineers in charge of evacuation plans for several European high schools;

- a security expert for chemical and fire risks within a European Civil Protection;

- An engineer in charge of evacuation procedures for a European railway company. 
Evaluators have been interviewed for one hour in order to collect their opinions and suggestions about the set of lemmas already extracted during the quantitative evaluation. As support for performed interviews, we have developed a visualization tool that allows an intuitive navigation, selection of terms or relations and improve the efficiency of the evaluation activity (see Figure 9). Moreover, in this way we can present to evaluators a high number of lemmas to analyze, maintaining them focused with less effort. Once a term or a relation has been selected, evaluators can modify or delete it. Finally, we have asked them to think aloud in order to collect opinions and suggestions.

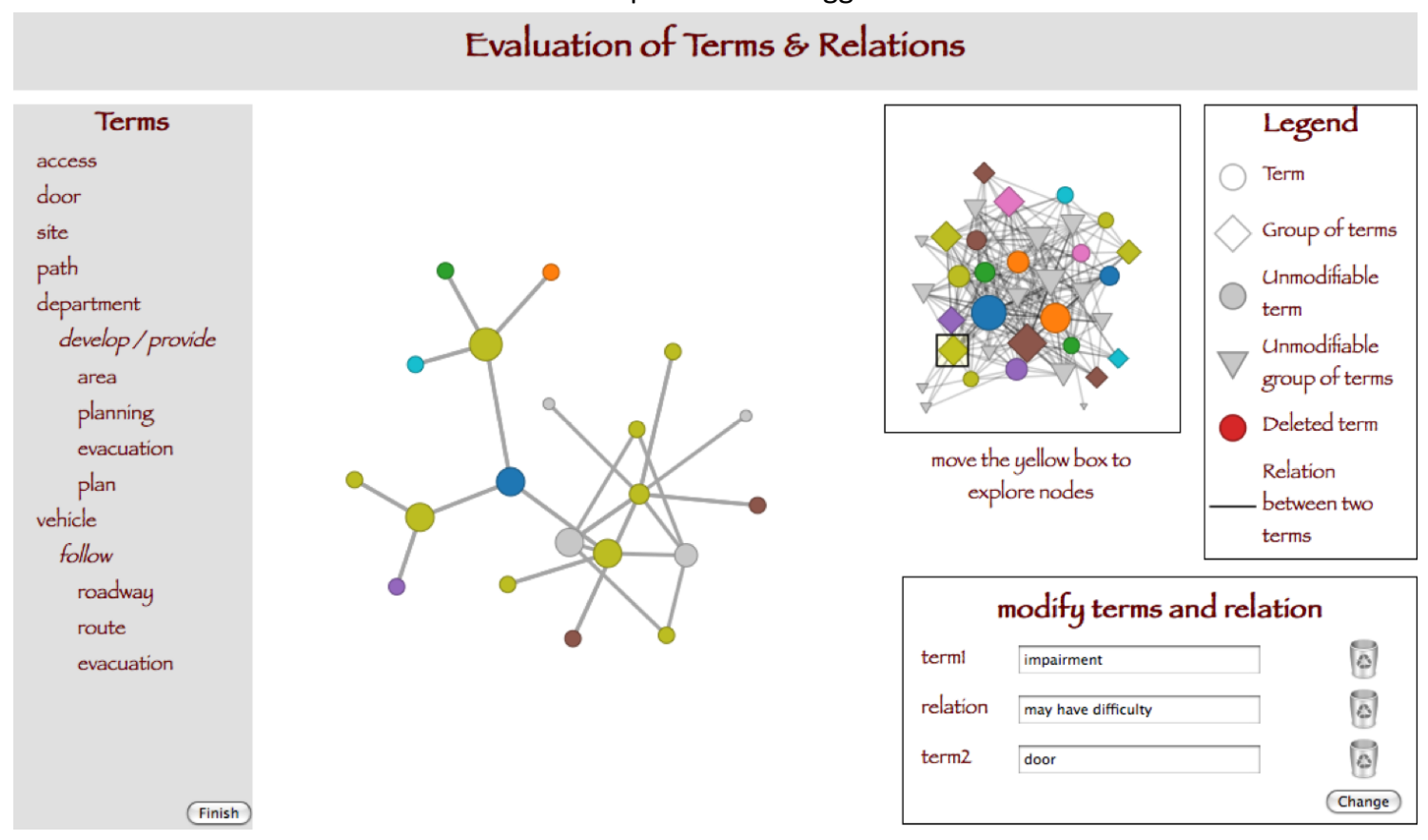

Figure 9.Visualization tool as support for the qualitative evaluation.

For each lemma, we have asked evaluators to take into account three main criteria (i.e. coverage, precision and accuracy) in form of the following questions: (1) Do you think the lemma belongs to the selected domain? (coverage); (2) Is the meaning of the lemma precise for the domain? (precision); (3) Does the lemma make sense for the domain? (accuracy). Results obtained for each evaluator are summarized in Table 2, while average values for coverage, precision and accuracy are presented in Figure 10 as a bar graph.

\begin{tabular}{|l|l|l|l|l|l|}
\hline & evaluator 1 & evaluator 2 & evaluator 3 & evaluator 4 & evaluator 5 \\
\hline Coverage & $98.36 \%$ & $97.70 \%$ & $90.48 \%$ & $97.87 \%$ & $97.87 \%$ \\
\hline Precision & $95.89 \%$ & $95.24 \%$ & $87.03 \%$ & $95.57 \%$ & $89.00 \%$ \\
\hline Accuracy & $92.78 \%$ & $92.28 \%$ & $86.70 \%$ & $94.42 \%$ & $93.00 \%$ \\
\hline
\end{tabular}

Table 2.The percentages for coverage, precision and accuracy for each evaluator. 


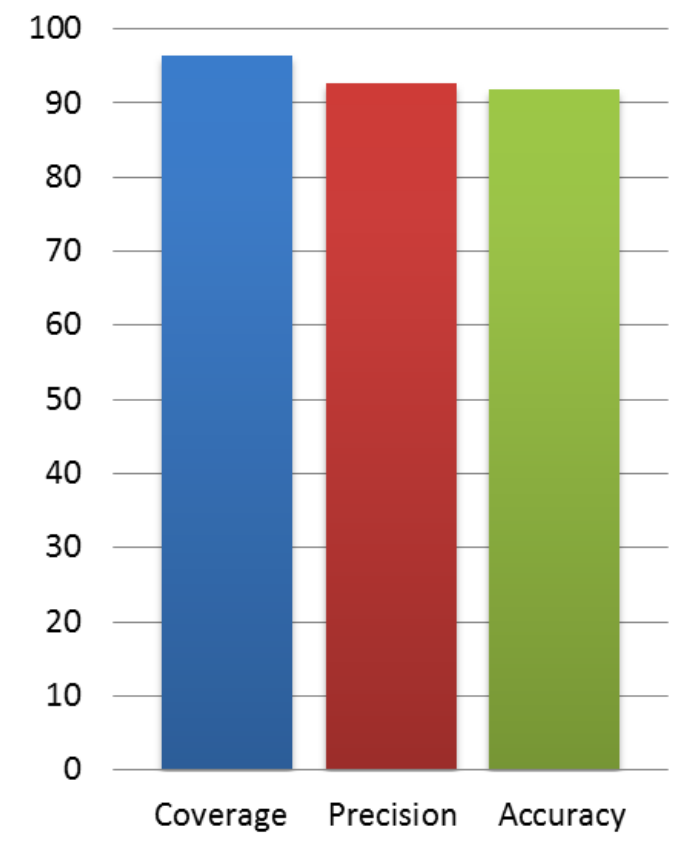

Figure 10. Average values for coverage, precision and accuracy. Each one of them is the percentage of the answers collected from the experts.

Values of coverage, precision and accuracy are all above the $85 \%$ and from a knowledge engineering point of view these are very good results that confirm the effectiveness of applied techniques for extracting terms and relations and computing their relevance.

Results collected from interviewed evaluators have been also used for improving the ontology, filtering out what was discarded by experts in terms of candidate terms and relations. In fact, after the qualitative evaluation we have performed a second iteration of quantitative evaluation using the same methodology already described in the previous section: definition of frequency classes taking into account the Zipf's law and filtering out what suggested by experts.

Considering the same frequency classes FC identified during the first iteration $(F C<28,28 \leq F C<500$, FC $\geq 500$ ), we have computed both coverage and accuracy obtaining a significant improvement: $76.9 \%$ for accuracy $(64.2 \%$ in the first iteration) and $65.4 \%$ for coverage (58.9\%). Figure 11 shows a graphic of the improved accuracy with the frequency classes on the horizontal axis and the number of lemmas on the vertical axis. The accuracy is represented by the percentage occupied by the overlapping area between the ontology part (i.e. pink) and the corpus part (i.e. brown).

As confirmed by such improvement, the qualitative filtering performed by domain experts has reduced terms that were less specific or quite general or reduced ambiguous relations, improving the representative value of final version of the ontology.

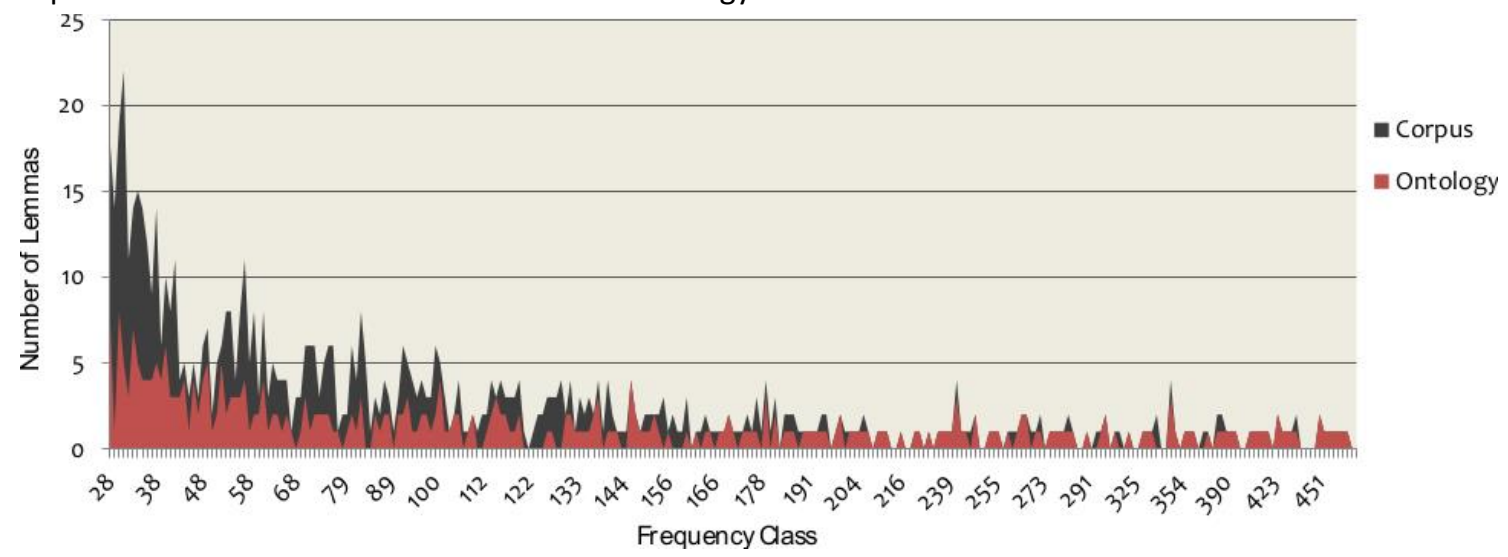

Figure 11. Second iteration of the accuracy of the ontology over the corpus 


\section{Conclusions}

During an emergency, involved organizations and agencies aim to reduce the number of victims and damages. Providing people with useful information about nearest safe places and routes to reach them can help in approaching the proposed objective. Moreover, it is important to guarantee that each user can escape from his/her location using provided information. For this reason, evacuation routes should be flexible and adaptable depending, not only on characteristics related to emergencies and affected areas, but also on user's profiles. It is crucial to take into account the abilities and special needs of affected people that could be related not only to functional but also situational disabilities. For example, in case of a fire event the smoke could affect the visibility of the environment and consequently the users could have a visual impairment to take into account for alerting and guiding them adequately.

In the background section, we have presented a list of aspects that every evacuation system, tool or model has to consider in order to provide an effective evacuation route diagram. In particular, by reviewing current systems we have found out that there is an evident lack in interoperability but also in automatically adapting the evacuation routes depending on users, emergency scenario and any exceptional circumstance.

The solution we have proposed in this paper is to extend an existing ontology, called SEMA4A. The SEMA4A ontology has been implemented to give a knowledge representation for accessible emergency notifications, focusing on three different domains: accessibility guidelines, emergency and communication technologies. The main idea of this work was to adapt provided alerts depending on abilities and expertise of affected users, characteristics of the emergency and available communication infrastructure. Our proposal is to integrate a fourth domain about evacuation routes for deriving information about adaptable and accessible evacuation routes.

The extended version of SEMA4A represents a valuable contribution from both a practical and theoretical point of view. As shown in the use case, the ontology can be used as basis for developing accessible notification systems. The iNERES application is a practical example of how the personalization of crucial information like instructions for escaping from a dangerous location can be automatically inferred from SEMA4A. Moreover, the integration of the ontology with any system and in any platform is made easier by the standard language OWL.

As shown briefly in the background section, current literature lacks of works including the four domains of interest considered in this work: accessibility, technology, emergency and evacuation. Consequently, from a theoretical point of view SEMA4A contributes to the research community for two main reasons. First of all, it can be considered as a knowledge base for future works in related research areas. Secondly, among known techniques for building ontologies we have applied a methodology specific for the kind of documents available in the evacuation domain that might be used in future applications.

Finally, in order to validate the extension of SEMA4A, we have performed a quantitative evaluation based on computing two measures, coverage and accuracy and a qualitative evaluation involving several experts in evacuation procedures. Moreover, we have used results obtained from experts to filter candidate terms and relations, reaching almost a ratio of $77 \%$ for the SEMA4A accuracy. This is related to two different aspects: technical and specific documents selected for the corpus and applied methodology for the information extraction.

As shown in (Manley and Kim, 2012), the personalization of evacuation routes for vulnerable groups require to take into account several factors, as for example the need of special tools or the interaction with other people in the same building. In this paper, we have presented a use case where the performed evaluation involveed users unfamiliar with the environment as an example of contextual disability. In future, it would be interesting to involve different kinds of vulnerable groups or more complex users' profiles in order to exploit all the practical implications given by SEMA4A.

Another topic addressed here is the definition of an interoperable knowledge model for integrating different domains. In these last years, there is a growing interest towards the application of ontologies as common basis for different kinds of expert systems. For example, in (Su et al., 2012) authors present 
a system for medical rehabilitation based on the definition of an ontology. The ontology is used as a formal language for inferring information for both patients and people interested in knowing more about the medical rehabilitation. Similar to this work but but applied to another domain is the work introduced by Neves et al. (Neves et al., 2014). In this case, the ontology is used not only as common language for managing information but also for guaranteeing the personalization depending on the environment and the users' preferences.

The personalization of critical information and the definition of knowledge models are the research directions we intend to follow for future contributions. On the one hand, we are going to work on the development of more complex use cases and practical applications based on SEMA4A. On the other hand, we are planning to perform a more extensive qualitative evaluation. The idea is to involve experts within international organisms, such as schools, hospitals or stadiums that have an extensive experience in developing evacuation routes.

\section{Acknowledgments}

This work has been partly funded by TIPEx (Ministerio de Economía y Competitividad TIN2010-19859C03-01), EmergenSys (Ministerio de Economía y Competitividad IPT-2011-1532-430000) and Antastic (Research Council of Norway - 220050/F11) projects.

\section{References}

Aedo, I., Yu, S., Díaz, P., Acuña, P., and Onorati, T. (2012). Personalized alert notifications and evacuation routes in indoor environments. Sensors (Basel), 12 (6): 7804-27.

Hamacher, H. W., and Tjandra, S. A. (2001) Mathematical Modeling of Evacuation Problems: A State of Art. Pedestrian and Evacuation Dynamics, 24 (24): 227-266.

Kim, S., George, B., and Shekhar, S. (2007) Evacuation route planning: scalable heuristics.Proceedings of the 15th annual ACM international symposium on Advances in geographic information systems, Seattle, WA, USA.

Koo, J. Kim, Y.S., Kim, B.I., and Christensen, K.M. (2013) A comparative study of evacuation strategies for people with disabilities in high-rise building evacuation, Expert Systems with Applications, 40 (2): 408 417.

Kotis, K., and Vouros, G. A. (2005).Human-centered ontology engineering: The HCOME methodology. Knowledge and Information Systems, 10 (1): 109-131. Springer.

Li, X., Liu, G., Ling, A., Zhan, J., An, N., Li, L., and Sha, Y. (2008). Building a PracticalOntology for Emergency Response Systems. 2008 International Conferenceon Computer Science and Software Engineering, 4:222-225.

Lozano-Tello, A., Macías, M., Prieto, A., Sánchez, F., and Sosa, E. (2004). ContenidosWeb Accesibles a Invidentes Meditante Ontologías. In II Congreso VirtualDerecho y Discapacidad en el Nuevo Milenio, Cáceres.

Luhn, H. P. (1958) The Automatic Creation of Literature Abstracts. IBM Journal of Research and Development, 2 (2): 159-165.

Maier, R. (2007) Knowledge Management Systems: Information and Communication Technologies for Knowledge Management. Volume 2.Springer.

Malizia, A., Acuna, P., Onorati, T., Díaz Pérez, P., and Aedo, I. (2009) CAP-ONES: an emergency notification system for all. International Journal Of Emergency Management, 6 (3/4): 302-316.

Malizia, A., Onorati, T., Díaz Pérez, P., Aedo, I., and Astorga-Paliza, F. (2010) SEMA4A: An ontology for emergency notification systems accessibility. Expert Systems with Applications, 37 (4): 3380-3391. 
Manley, M., and Kim, Y.S. (2012) Modeling emergency evacuation of individuals with disabilities (exitus): An agent-based public decision support system, Expert Systems with Applications, 39 (9): 8300-8311.

Miller, G. A., Beckwith, R., Fellbaum, C., Gross, D., and Miller, K. J. (1990) Introduction to WordNet: An On-line Lexical Database. International Journal of Lexicography, 3 (4): 235-244.

Navigli, R., Velardi, P., and Gangemi, A. (2003) Ontology Learning and its Application to Automated Terminology Translation. IEEE Intelligent Systems, 18: 22-31.

Neves, A. R. D. M., Carvalho, A. M. G., and Ralha, C. G. (2014) Agent-based architecture for contextaware and personalized event recommendation. Expert Systems with Applications 41 (2): 563-573.

Spyns, P., andReinberger, M-L. (2005) Lexically Evaluating Ontology Triples Generated Automatically from Texts. The Semantic Web: Research and Applications (Gómez-Pérez A, Euzenat J, eds.), 3532.

Su, C. J., and Peng, C. W. (2012)Multi-agent ontology-based Web 2.0 platform for medical rehabilitation. Expert Systems with Applications 39 (12): 10311-10323.

Wagner, N., and Agrawal, V. (2014) An agent-based simulation system for concert venue crowd evacuation modeling in the presence of a fire disaster, Expert Systems with Applications, Volume 41, Issue 6, May 2014, 2807-2815.

Yamada, T. (1996) A network flow approach to a city emergency evacuation planning. International Journal of Systems Science, 27 (10): 931-936.

Yesilada, Y., Harper, S., Goble, C., and Stevens, R. (2004). Screen Readers CannotSee (Ontology Based Semantic Annotation for Visually Impaired Web Travellers).Web Engineering 4th International Conference ICWE 2004 Proceedings LNCS 3140: 445-458.

Zipf, G. K. (1949) Human Behaviour and the Principle of Least-Effort. Addison-Wesley. 